Journal of Fundamental and Applied Sciences

ISSN 1112-9867

Available online at

http://www.jfas.info

\title{
PREVALENCE AND ANTIBIOTIC RESISTANCE OF STAPHYLOCOCCI ISOLATED FROM KOLEA HOSPITAL (ALGERIA)
}

\author{
M. N. Boukhatem ${ }^{1}$, M. A. Ferhat ${ }^{2}$, R. Hadj Mohamed ${ }^{1}$, N. Lalaoui ${ }^{3}$ \\ ${ }^{1}$ Département de Biologie et Physiologie Cellulaire, Faculté des Sciences de la Nature et de la Vie, \\ Université Blida 1, Algeria. \\ ${ }^{2}$ Département de Chimie, Ecole Normale Supérieure de Kouba, Alger, Algeria. \\ ${ }^{3}$ Laboratoire de Bactériologie, Etaplissement Public Hospitalier de Koléa, Tipaza, Algeria.
}

Received: 16 February 2015 / Accepted: 07 March 2015 / Published online: 09 March 2015

\begin{abstract}
The resurgence of multiple antibiotic resistances is a major public health problem, often causing therapeutic impasses. Our study was conducted at the Bacteriology Laboratory of Kolea (Tipaza, Algeria) Hospital. The aim of this study was to determine the prevalence of staphylococci in clinical purulent, sepsis and urinary samples, and to test the sensitivity of the selected strains against a various antibiotic (ATB) discs. The identification of Staphylococcus strains was performed by using classic biochemistry tests. Susceptibility against ATB was determined by the agar disk diffusion method. Among 1403 clinical samples obtained, only 61 was related to staphylococci infections (4.34\%). Staphylococcus strains were found mainly in the purulent samples (60.65\%). S.aureus was found to be the mainly isolated strains with a rate of $61.53 \%$ while the S. epidermidis strain was found predominantly in hospitalized patients $(68.57 \%)$. The frequency of the overall resistance of S.aureus against penicillin $\mathrm{G}(80.6 \%)$ and gentamicin $(61.53 \%)$ remains high. The multi-resistant Staphylococcus has become extremely widespread, hence the need for the accomplishment of a strategy to prevent the spread of this resistance.
\end{abstract}

Keywords: Staphylococci; Staphylococcus aureus; Oxacillin; Antibiotic resistance; Disc diffusion.

Author Correspondence, e-mail: mn.boukhatem@yahoo.fr

ICID: 1142924 


\section{INTRODUCTION}

L'évolution rapide de la résistance bactérienne aux antibiotiques (ATB) est un phénomène actuellement préoccupant dans les pays en voie de développement où les pathogènes résistants peuvent avoir une plus forte prévalence dans certains pays africains. L'Algérie est un pays du nord de l'Afrique où les récentes données de résistance aux ATB indiquent une situation inquiétante. En effet, ces dix dernières années ont été marquées par l'émergence et la dissémination de nouveaux gènes de résistance notamment dans le nord du pays [1].

Les espèces du genre Staphylococcus figurent parmi ces germes qui ont un fort pouvoir adaptatif et ont développé différents mécanismes de résistance aux ATB. Plus de 90\% des souches produisent une pénicillinase. L'oxacilline reste active contre ces souches, mais des staphylocoques hospitaliers ont développé une résistance croisée entre les pénicillines $M$ (méticilline, oxacilline) et les autres $\beta$-lactamines [2]. De plus, la pathogénéicité des staphylocoques pose peu de problème en ce qui concerne Staphylococcus aureus. Ce dernier est à l'origine de pathologies extrêmement variées, qui peuvent être des infections suppuratives, localisées ou systémiques. Ces infections relèvent d'un véritable problème de santé publique, tant par la virulence de la bactérie que par l'émergence de souches multirésistantes. Selon les services hospitaliers, ces dernières représentent entre 20 à $50 \%$ des souches [3]. Les premiers cas rapportés d'infection à S. aureus résistant à la méticilline (SARM) datent de plus de trente ans et revêtaient un caractère nosocomial, l'acquisition de SARM étant liée à l'hospitalisation récente ou à l'exposition prolongée et récurrente aux ATB. Face à cette préoccupation mondiale - qui est l'émergence des staphylocoques multi-résistants -, il est primordial de conduire des études épidémiologiques afin de comprendre et de contrôler la diffusion et l'augmentation de la résistance des staphylocoques aux ATB [4]. C'est dans ce contexte que nous avons orienté notre étude qui a été réalisée au niveau du laboratoire de bactériologie de l'hôpital Koléa (Tipaza, Agérie). Notre travail s'est axé principalement sur le diagnostic bactériologique des prélèvements purulents, urinaires et septicémiques, provenant de patients hospitalisés ou des consultations externes, en vue de déterminer la fréquence des staphylocoques et d'évaluer ainsi leur profil de résistance aux ATB, afin de mieux définir les stratégies thérapeutiques et préventives.

\section{MATERIEL ET METHODES}

\subsection{Milieux de culture et solutions de coloration :}


Plusieurs milieux de culture ont été utilisés lors de notre étude. Nous citons la gélose nutritive (GN), gélose Muller-Hinton (MH) ou encore le milieu Chapman. Tous ces milieux de culture proviennent de l'Institut Pasteur d'Alger (IPA). Concernant les solutions de colorations utilisées, nous citons le violet de gentiane, lugol, fuchsine et alcool $\left(70^{\circ}\right)$. Toutes ces solutions ont été utilisées pour la réalisation de la coloration de Gram des bactéries isolées.

\subsection{Tests et réactifs d'identification :}

L'identification des souches de staphylocoques isolées a été faite à l'aide d'une mini-galerie classique. Les milieux et réactifs utilisés sont : Triple Sugar Iron (TSI), milieu Mannitol mobilité, bouillon nitraté, réactif N1 (acide sulfanilique) et N2 ( $\alpha$-naphtylamine), plasma humain citratée, Lysine décarboxylase (LDC), Ornithine décarboxylase (ODC), Arginine dihydrolase (ADH) et poudre de Zinc.

\subsection{Diagnostic bactériologique et antibiogramme}

Notre étude a été effectuée au niveau du laboratoire de Bactériologie de l'EPH de Koléa (wilaya de Tipaza, Algérie), durant une période de 04 mois (Mai-Août 2014). L'objectif de notre travail consiste à rechercher et identifier les espèces appartenant au genre Staphylococcus, isolées principalement des prélèvements purulents, urinaires ou encore ceux destinés à l'hémoculture. Ces prélèvements ont été diagnostiqués dans les différents services de l'EPH Koléa. Aussi, des prélèvements de patients, auscultés à titre externe, ont été obtenus. En fonction des résultats de la coloration de Gram, le prélèvement a été ensemencé sur un milieu Chapman. L'identification de l'espèce a été faite en se basant sur les caractères morphologiques, culturaux et biochimiques. Par la suite, un antibiogramme, par la méthode de diffusion de disque sur gélose Mueller-Hinton (MH), a été réalisé sur chaque espèce bactérienne. Les disques utilisés proviennent du laboratoire bio-Mérieux (France). La lecture et l'interprétation des résultats ont été faites selon les règles et les recommandations du Comité d'Antibiogramme de la Société Française de Microbiologie [5].

\section{RESULTATS}

\subsection{Fréquence des prélèvements après culture microbienne:}

Sur un total de 1403 prélèvements analysés au laboratoire, une grande majorité (74.83\%) a été déclarée exempte de toutes contaminations microbiennes. Par ailleurs, les cultures retrouvées positives représentent une fréquence de $22.73 \%$ alors qu'un faible taux $(2.42 \%)$ a été enregistré 
comme étant des cultures contaminées. Ce faible nombre témoigne du respect des conditions d'asepsie lors de l'analyse bactériologique.

\subsection{Répartition des cultures positives selon le prélèvement :}

$\mathrm{Au}$ total, 319 prélèvements ont été déclarés comme présentant une croissance microbienne. La majorité sont issus de prélèvements urinaires, soit un nombre de 119 ce qui représente un taux de $62.38 \%$, suivi par les prélèvements purulents avec un taux de $31.03 \%$ et, enfin, les prélèvements sanguins qui ne représentaient que 21 cas soit un taux de $06.58 \%$.

\subsection{Fréquence des staphylocoques dans tous les prélèvements :}

Après avoir effectués les examens cytobactériologique des pus et des urines ainsi que les hémocultures, il en ressort que les souches staphylococciques ont été incriminées dans 61 de cas des infections humaines. Ces souches ont été trouvées prédominantes dans les prélèvements purulents des plaies (Fig.1) (superficielles ou profondes) avec un taux de 60.65\%, suivis par les prélèvements urinaires et enfin ceux destinés à l'hémoculture.

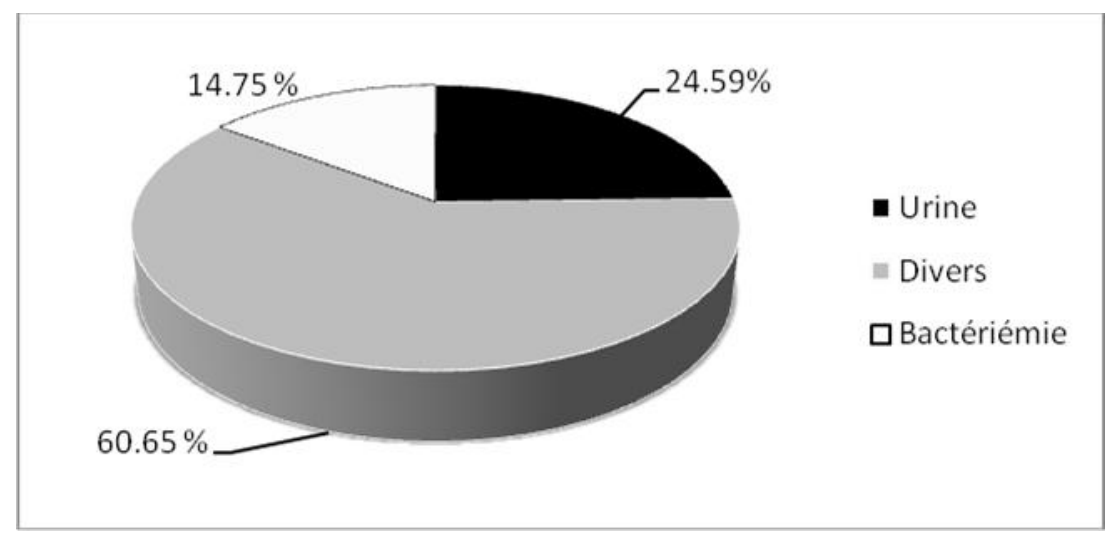

Fig.1. Fréquence des staphylocoques dans tous les prélèvements.

\subsection{Répartition des staphylocoques selon le sexe :}

Il en ressort, des résultats obtenus, que le nombre des staphylocoques isolés des prélèvements analysés est presque similaire chez les deux sexes, avec néanmoins une légère prédominance masculine (57.37\%). Le sexe-ratio est de 0.79 .

\subsection{Répartition des staphylocoques selon l'espèce isolée :}

Une fois les colonies présumées de staphylocoques ont été isolées sur milieu Chapman, une identification morphologique et biochimique a été effectuée afin de déterminer l'espèce bactérienne incriminée. Il apparait clairement que les isolats de S. epidermidis sont ceux qui ont été le plus souvent isolés avec un taux de $57.37 \%$, alors que les staphylocoques pathogènes, représentés par la 
souche de $S$. aureus, ont été incriminées dans $42.62 \%$ des cas. En effet, l'espèce de $S$. aureus a pour capacité de dégrader le mannitol du milieu Chapman et la réduction du nitrate. Elle se caractérise par la fermentation de glucose, de lactose et de saccharose. Elle a aussi un caractère pathogène qui permet de coaguler le sérum humain. Par ailleurs, S. epidermidis se caractérise par l'absence de la coagulase et la non dégradation du mannitol (Tableau 1).

Tableau 1. Principaux caractères biochimiques des staphylocoques isolés.

\begin{tabular}{lccccc}
\hline Espèce & TSI & Nitrate réductase & Mannitol & ADH & Coagulase \\
\hline S. aureus & + & + & + & + & + \\
S. epidermidis & + & + & - & - & - \\
\hline
\end{tabular}

TSI : Tri Sugar Iron; ADH : Arginine Dihydrolase ; (+) Réaction positive ; (-) réaction négative.

\subsection{Répartition des espèces de staphylocoques selon les services :}

Eu égards des résultats obtenus (Fig.2), il apparait clairement les staphylocoques pathogènes ont été les plus incriminés dans les infections communautaires du moment que 16 prélèvements positifs ont été enregistrés. En revanche et concernant les prélèvements provenant de patients hospitalisés, c'est principalement les Staphylocoques Coagulase Négative $(\mathrm{SCN})$ qui ont été retrouvés avec une fréquence élevée (24 cas). Ceci pourra être expliqué par le fait que certains patients ont été déjà soumis à une antibiothérapie, notamment pour les prélèvements des patients présentant une septicémie. Cette antibiothérapie est à l'origine de la réduction des isolats staphylococciques pathogènes ; uniquement les germes saprophytes pourront alors être cultivés.

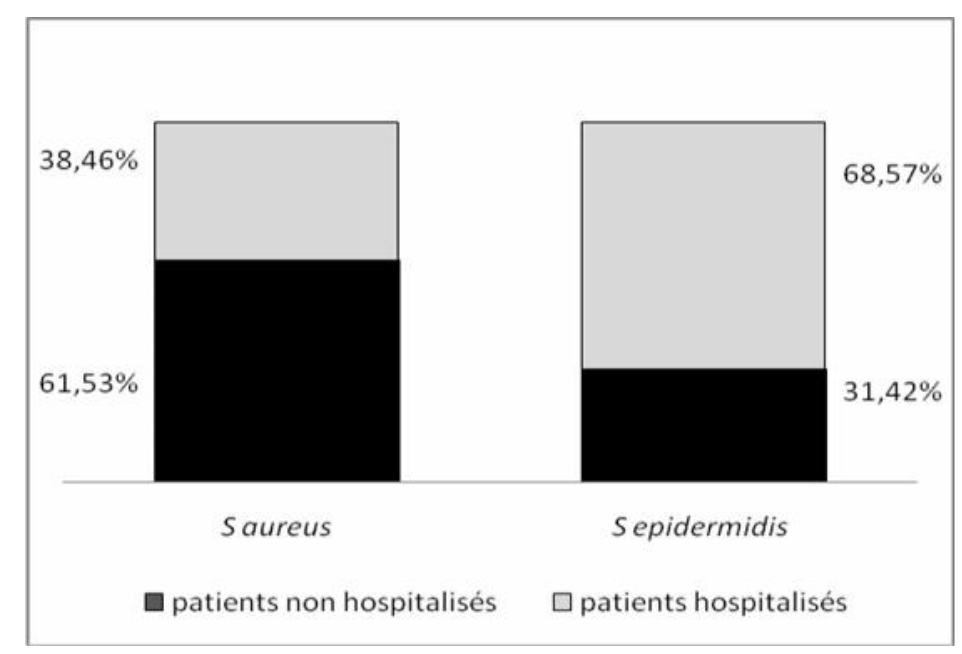

Fig.2. Répartition des espèces staphylococciques selon la provenance des prélèvements. 


\subsection{Antibiorésistance des souches staphylococciques isolées}

Une fois les étapes d'isolement et d'identification des souches staphylococciques ont été terminées, une étude du profil d'antibiorésistance de ces isolats a été effectuée en utilisant la technique de diffusion à partir de disque.

\subsubsection{Profil de résistance des Staphylococcus aureus :}

Toutes les souches de $S$. aureus ont été soumises à un antibiogramme pour vérifier leur résistance vis-à-vis de 11 ATB appartenant à différentes familles. Les résultats de cette analyse sont rapportés dans la Figure 3. Les résultats obtenus montrent une grande résistance des $S$. aureus vis-à-vis de la pénicilline avec un taux de $84.61 \%$, suivie de la gentamycine $(61.53 \%)$ et la kanamycine $(57.92 \%)$. En revanche la vancomycine et la pristinamycine restent parmi les ATB les plus efficaces car aucune résistance n'a été notée pour les souches staphylococciques pathogènes.

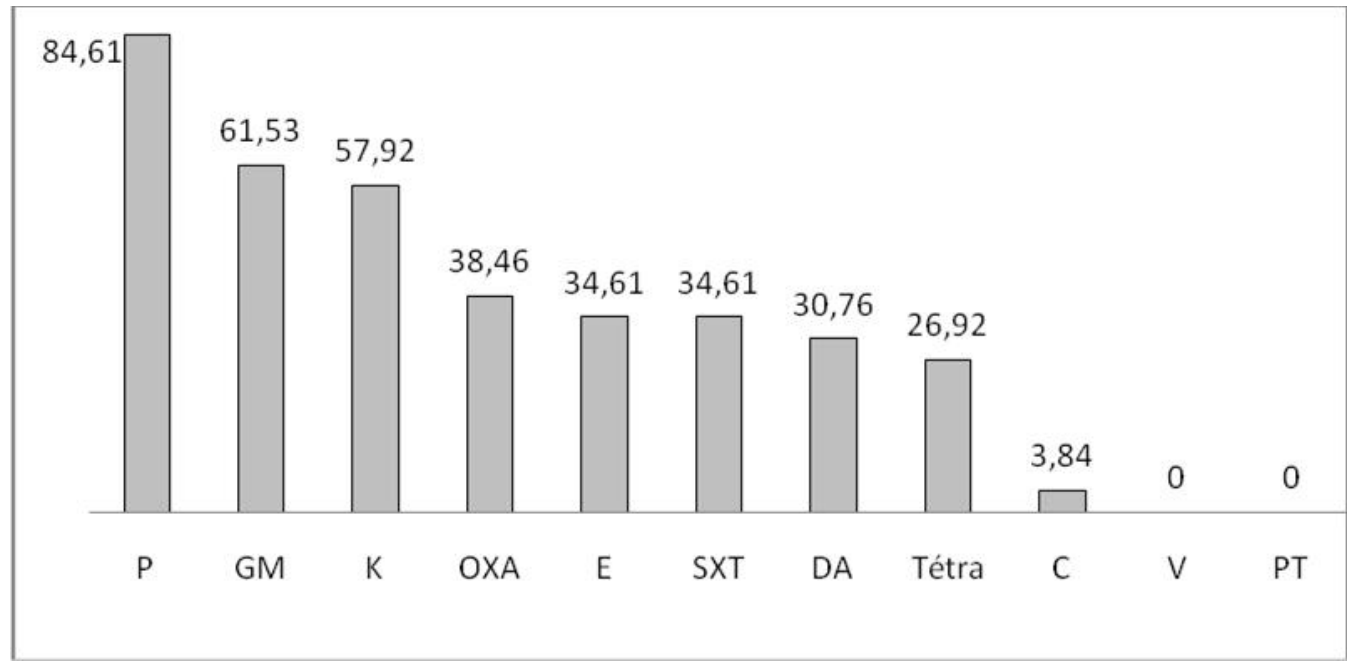

Fig.3. Profil de résistance des Staphylococcus aureus.

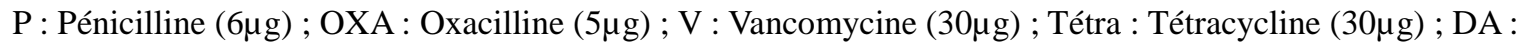

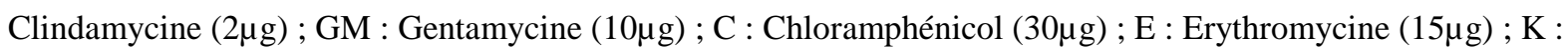
Kanamycine $(30 \mu \mathrm{g})$; PT : Pristinamycine $(15 \mu \mathrm{g})$; SXT : Cotrimoxazole $(15 \mu \mathrm{g})$.

\subsubsection{Profil de résistance des Staphylococcus epidermidis :}

Concernant les souches SCN, le profil de cette antibiorésistance est rapporté dans la Figure 4. Dans le même sillage que celui des $S$. aureus, les souches de $S$. epidermidis sont résistantes à la pénicilline avec un taux très élevé $(82.85 \%)$, suivies par le cotrimoxazole $(68.57 \%)$. En revanche, aucune résistance n'a été enregistrée pour le chloramphénicol et la pristinamycine. 


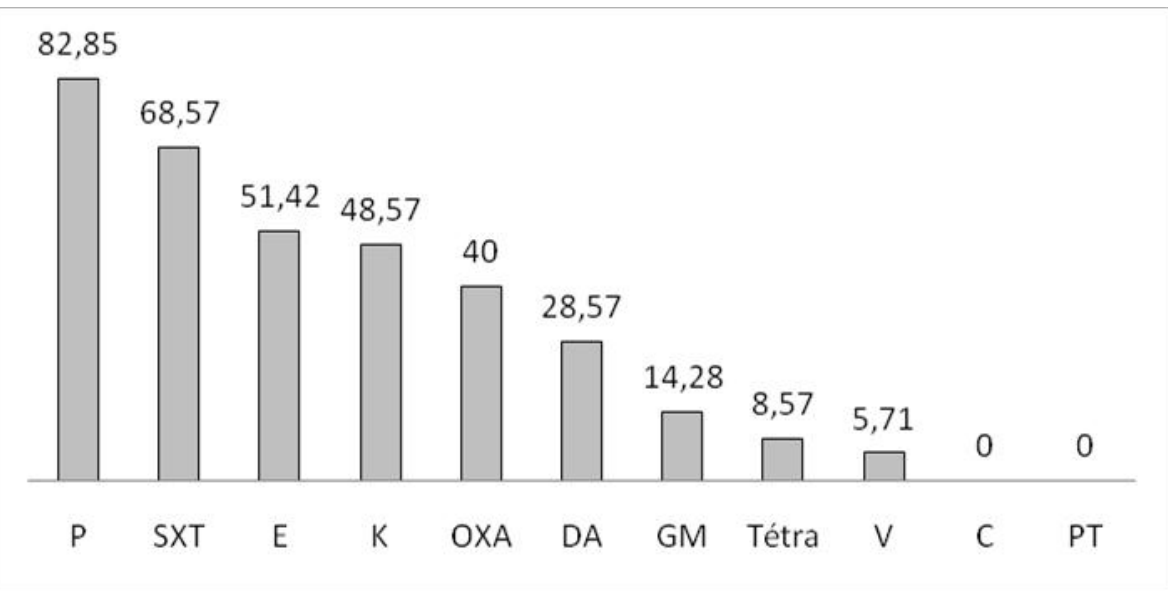

Fig.4. Profil de résistance des Staphylococcus epidermidis isolés.

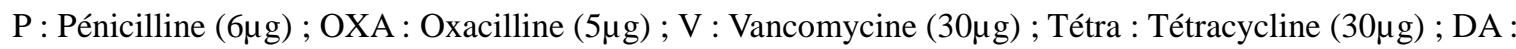

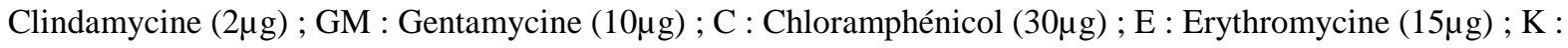

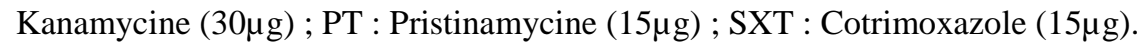

\section{DISCUSSION}

Notre travail s'est axé principalement sur le diagnostic bactériologique des prélèvements purulents, urinaires et ceux destinés à l'hémoculture, en vue de déterminer la fréquence des espèces du genre Staphylococcus isolées et d'évaluer ainsi leur profil de résistance aux ATB, afin de mieux définir les stratégies thérapeutiques et préventives. Notre étude répond à cet objectif par l'analyse de l'activité, in vitro, de plusieurs ATB contre 26 souches de $S$. aureus et 35 de $S$. epidermidis provenant des prélèvements sus-indiqués au niveau de l'hôpital de Koléa (Tipaza). En effet, la connaissance et la surveillance du profil de sensibilité des staphylocoques sont primordiales dans la prise en charge des infections générées par ces espèces bactériennes, ainsi que la maîtrise de leur diffusion clonale. Les prélèvements urinaires occupent aussi une place privilégiée où les staphylocoques ont été incriminés dans près de $1 / 4$ de cas. Le pourcentage des uro-infections à staphylocoques dans cette étude $(4.59 \%)$ peut être très difficilement comparé, du fait d'un manque de données dans la littérature. Une plus grande fréquence de staphylocoques est isolée en Allemagne : $20.6 \%$ des malades hospitalisés et ils prennent la troisième place des uropathogènes après les entérocoques (29\%) et E. coli (22\%), d'après les résultats d'une étude pendant la période de 1990-1991 [6]. Il y a une différence dans les proportions entre $S$. aureus et CNS. Chez les malades étudiés qui souffraient d'uro-infections compliquées ou nosocomiales, S. aureus existe chez $31 \%$ et 
les $\mathrm{SCN}$ - chez $69 \%$, tandis que le pourcentage de ces derniers est considérablement plus grand : $86,5 \%$ CNS et $13,5 \%$ S. aureus [6].

Malgré le fait que le type S. aureus est plus rarement isolé dans les uro-infections, son importance comme agresseur des papilles rénales est très connue. On accepte que la colonisation précède l'infection staphylococccique, comme Archer [7], qui nous signale qu'à peu près $30 \%$ des personnes saines sont colonisées au S. aureus. La porte d'entrée est avant tout nasale, mais aussi vaginale et péri-anale $[7,8]$.

La souche $S$. aureus a été retrouvée prédominante dans les prélèvements des infections communautaires avec une fréquence de $61.53 \%$ (16 isolats) comparativement aux prélèvements provenant de patients hospitalisés où un taux de $38.46 \%$ a été enregistré. Cette tendance a été inversée concernant les SCN où 24 isolats ont été isolés représentant un taux de $68.57 \%$. Nos résultats ont été corroborés par les données de la littérature. Les infections à staphylocoques sont ubiquitaires et peuvent se présenter sous forme d'infections communautaires ou surtout sous forme d'infections nosocomiales. L'épidémiologie des infections à staphylocoques évolue, au cours de la vie, et passe quasi-obligatoirement par un portage essentiellement nasal antérieur. Certains patients ont un risque plus élevé de colonisation nasale à staphylocoque : diabétiques insulinodépendants, dialysés chroniques, toxicomanes par voie veineuse, patients porteurs du VIH ou sida déclaré, patients âgés en maison de retraite médicalisée $[9,10]$.

L'information pour la prévalence des SCN des urines, par d'autres auteurs [6] a été confirmée sur nos isolats. La haute fréquence des SCN est liée avant tout à l'hospitalisation. On accuse le rôle du milieu hospitalier pour la dissémination de telles souches [11,12].

Staphylococcus aureus a un fort pouvoir adaptatif et a développé différents mécanismes de résistance aux antistaphylococciques. Plus de $90 \%$ des souches produisent une pénicillinase. L'oxacilline reste active contre ces souches, mais des staphylocoques hospitaliers, et plus récemment communautaires (présents hors de l'hôpital), ont développé une résistance croisée entre les pénicillines $\mathrm{M}$ (méticilline, oxacilline) et les autres $\beta$-lactamines [2]. Ces résultats ont été confirmés lors de notre étude où nous avons noté que les isolats staphylococciques, pathogènes ou non, demeurent très résistants aux ATB de la famille des $\beta$-lactamines, en particulier la pénicilline. La fréquence de la résistance globale des $S$. aureus, hospitalières et communautaires, à la pénicilline G $(80.6 \%)$ et gentamycine $(61.53 \%)$ demeure élevée. Des résultats similaires aux nôtres ont été 
rapportés par Lowy [13] et Elhamzaoui [4] qui indiquent qu'actuellement plus de $90 \%$ des souches de $S$. aureus sont résistantes à la pénicilline $\mathrm{G}$ par production de pénicillinase.

Dans notre étude, la Pénicilline G reste l'antibiotique le moins actif vis-à-vis des souches pathogènes ou non. Cette tendance a été confirmée en différents points du monde par plusieurs études antérieures [14].

Par ailleurs et eu égards des résultats obtenus lors de notre étude, il a été constaté que le taux de résistance à l'oxacilline chez $S$. aureus est de $38.46 \%$, taux plus élevée que celui trouvé dans les cinq principaux hôpitaux du district de Thessalie (Grèce centrale) puisque $14.8 \%$ des isolats étaient résistants [14] et plus élevé encore que dans les pays nordiques (Suède, Danemark) où le pourcentage de $S$. aureus résistant à l'oxacilline est resté très bas $(<2 \%)$ [4]. En outre, nous retrouvons dans notre étude les mêmes résultats que ceux obtenus par Lelièvre [15], montrant une plus grande sensibilité parmi les souches sensibles à la gentamicine, à la rifampicine et au cotrimoxazole.

Par ailleurs, vancomycine et pristinamycine demeurent les ATB les plus actives sur la totalité des souches S. aureus (100\%), contrairement à Garnier [16], qui a retrouvé une plus grande résistance dans un centre pédiatrique de la région parisienne. La même constatation a été faite par Ravaoarinoro et Therrien [17] qui ont démontré que la teicoplanine et la vancomycine sont deux à huit fois plus actives que les autres ATB testés contre la majorité des staphylocoques, en particulier les SARM. Rappelant que la vancomycine a été sur le marché depuis 30 ans et est le médicament de choix pour le traitement des infections graves causées par les bactéries à Gram positif dans de nombreux hôpitaux, toutefois, son utilisation abusive a aboutit ces dernières années, à l'apparition de résistance très élevée chez les staphylocoques à coagulase négative (de 65 à 80 \%) même dans les pays nordiques (de 30 à $40 \%)[4,18]$.

Le gentamycine est un produit, qui est proposé par certains auteurs pour le traitement des infections à staphylocoques [16], mais nos résultats ont prouvé que pour ce produit existent des différences importante concernant la sensibilité entre les souches S. aureus et les souches S.epidermidis. Les $\mathrm{SCN}$ réagissent bien à gentamycine avec un taux de $85.71 \%$, tandis qu'avec les souches pathogènes, cet ATB est actif uniquement sur 38.46\%. Un effet considérablement meilleur à été constaté avec un autre ATB de la même famille (aminosides), en l'occurrence la kanamycine, où parmi les SCN, $51.42 \%$ des isolats se sont manifestés sensibles. 


\section{CONCLUSION}

Les infections humaines dues aux staphylocoques occupent une place importante en pathologie infectieuse en raison de leur fréquence et de leur gravité, tant au niveau de l'hôpital qu'au sein des populations. La multi-résistance des staphylocoques est devenu remarquablement répandue dans les prélèvements humains, d'où la nécessité de la mise en œuvre d'une stratégie active et efficace garantissant une sécurité microbiologique afin d'éviter la propagation de cette résistance.

Les conséquences médicales (majoration de la mortalité) et économiques (durées de séjours plus longues, coûts des traitements) générées par ces infections ont déjà été largement évaluées. Cet état de fait justifie dans les établissements sanitaires la mise en place de moyens adaptés de prévention de transmission par application des précautions standards ou complémentaires de contact, de détection de ces résistances bactériennes, de connaissance approfondie de l'écologie locale et une implication particulière pluridisciplinaire dans la lutte contre les infections liées aux SARM.

\section{REFERENCES}

[1] Baba Ahmed-Kazi Tani Z., Arlet G., Pathol. Biol. 2014, 62(3), 169-178.

[2] Fernandez-Gerlinger M.P., Mainardi J.L., J. Anti-infect. 2014, 16(1), 18-23.

[3] Nuemi G., Astruc K., Aho S., Quantin C., Rev. Épidémiol. Santé. Pub. 2013, 61(5), 455-461.

[4] Elhamzaoui S., Benouda A., Elouennass M., Méd. Malad. Infect. 2009, 39(12), 891-895.

[5] Bonnet R., Cavallo J.D., Chardon H., Guery B., Com. Antibio. Soc. Fr. Microbiol. 2012.

[6] Guirguitzova B., Chankova D., Zozikov B., Ann. Urol. 2002, 36, 5, 341-347.

[7] Archer G. L., Clin. Infect. Dis.1998, 26(5), 1179-1181.

[8] Fabre R., Mérens A., Cavallo J.D., Ternois I., Pathol. Biol. 2013, 61(2), 44-48.

[9] Halablab M.A., Hijazi S.M., Araj G.F., Epidemiol. Infect. 2010, 138(05), 702-706.

[10] Stone N.D., Lewis D.R., Gaynes R.P., Infect. Contr. Hosp. Epidemiol. 2012, 33(6), 551-557.

[11]Huebner J., Goldmann D.A., Ann. Rev. Med. 1999, 50(1), 223-236.

[12] Otto M., Bioessays. 2013, 35(1), 4-11.

[13]Lowy F.D., J. Clin. Invest. 2003, 111(9), 1265-1273.

[14]De Sousa M.A., Bartzavali C., De Lencastre H., J. Clin. Microbiol. 2003, 41(5), 2027-2032.

[15]Lelièvre H., Lina G., Etienne J., J. Clin. Microbiol. 1999, 37(11), 3452-3457.

[16] Garnier F., Mariani-Kurkdjian P., Raymond J., Méd. Malad. Infect. 2002, 32(8), 432-438. 
[17] Ravaoarinoro M., Therrien C., Int. J. Antimicrob. Agen. 2013, 7(3), 167-170.

[18]David M.Z., Daum R.S., Clin. Microbiol. Rev. 2010, 23(3), 616-687.

\section{PREVALENCE ET ANTIBIORESISTANCE DES STAPHYLOCOQUES ISOLES AU NIVEAU DE L'HOPITAL DE KOLEA (ALGERIE)}

\section{RESUME}

La recrudescence de la multirésistance aux antibiotiques (ATB) constitue un problème majeur de santé publique, provoquant souvent des impasses thérapeutiques. Notre étude a été réalisée au laboratoire de bactériologie de l'hôpital Koléa (Tipaza, Algérie) sur des prélèvements purulents, urinaires et ceux destinés à l'hémoculture, provenant d'infections hospitalières ou communautaires, en vue de déterminer la fréquence des staphylocoques isolés et d'évaluer leur profil de résistance aux ATB. L'identification des souches a été réalisée avec une mini galerie classique. La sensibilité aux ATB a été déterminée par la méthode de diffusion des disques en milieu gélosé.

Sur 1403 prélèvements obtenus, 61 répondaient aux critères d'infection liée aux staphylocoques $(4.34 \%)$. Les isolats staphylococciques sont retrouvés essentiellement dans les prélèvements purulents $(60.65 \%)$. L'espèce Staphylococcus aureus domine le profil épidémiologique pour les infections communautaires avec un taux de $61.53 \%$ alors que la souche S. epidermidis a été retrouvée prédominante chez les patients hospitalisés $(68.57 \%)$. La fréquence de la résistance globale des S. aureus à la pénicilline G (80.6\%) et la gentamycine (61.53\%), demeure élevée. La multi-résistance des staphylocoques est devenu remarquablement répandue, d'où la nécessité de la mise en œuvre d'une stratégie efficace afin d'éviter la propagation de cette résistance.

Mots-clés : Staphylocoques; Staphylococcus aureus; Oxacilline; Résistance aux antibiotiques; Antibiogramme.

\section{How to cite this article}

Boukhatem M N, Ferhat M A, Hadj Mohamed R, Lalaoui N. Prevalence and antibiotic resistance of staphylococci isolated from kolea hospital (Algeria). J Fundam Appl Sci. 2015, 7(2), 260-270. 